\title{
Decomposition of group-valued measures on orthoalgebras
}

\author{
by
}

Paolo De Lucia (Napoli) and Pedro Morales (Sherbrooke, Qué.)

\begin{abstract}
We present a general decomposition theorem for a positive inner regular finitely additive measure on an orthoalgebra $L$ with values in an ordered topological group $G$, not necessarily commutative. In the case where $L$ is a Boolean algebra, we establish the uniqueness of such a decomposition. With mild extra hypotheses on $G$, we extend this Boolean decomposition, preserving the uniqueness, to the case where the measure is order bounded instead of being positive. This last result generalizes A. D. Aleksandrov's classical decomposition theorem.
\end{abstract}

1. Introduction. The initial impetus for the study of measures on nonBoolean orthostructures such as orthomodular lattices and orthomodular posets came from the logico-probabilistic foundations of quantum mechanics (see [3], [6], [18] and [24]). But the non-existence of a tensor product for orthomodular lattices or orthomodular posets (see [16, p. 264]) -necessary to describe coupled physical entities - has led to the introduction of orthoalgebras, a more general orthostructure a large class of which - called unital orthoalgebras - admits a tensor product (see [11]). Orthoalgebras are apparently one of the simplest and most natural orthostructures that can carry orthogonally additive measures, and today they provide a mathematical basis for the rapidly developing field of non-commutative measure theory (see [9], [10], [12]-[14], [20]-[22]).

In this paper we present a general decomposition theorem for a positive inner regular measure on an orthoalgebra $L$ with values in an ordered Hausdorff topological group $G$ not necessarily commutative. The generality of the context prevents of course the uniqueness of our decomposition. We show that it holds when $L$ is a Boolean algebra, getting what we call the First Decomposition Theorem. Moreover, adding some natural hypotheses on $G$

1991 Mathematics Subject Classification: 28B15, 46L50, 06F20.

The first author was supported by Ministero dell'Università e della Ricerca Scientifica e Tecnologica (Italy).

The second author was supported by NSERC of Canada. 
we can eliminate, in the Boolean case, the positivity restriction and give a unique decomposition of an order bounded inner regular measure on $L$ with values in $G$. This Second Decomposition Theorem generalizes the first classical Aleksandrov Decomposition Theorem [2, Theorem 3, pp. 618-619], and allows us, upon imposing a mild "topological" condition, to derive a Yosida-Hewitt decomposition of a $G$-valued order bounded inner regular finitely additive set function (see [25, Theorem 1.24]).

The paper is organized as follows. In Section 2, after the introduction of some pertinent subsets of $G^{L}$, we establish our general decomposition theorem and we deduce the First Decomposition Theorem. Section 3 defines the notion of topological lattice group satisfying the condition $(M)$ following Jameson [15] and formulates the Second Decomposition Theorem. Finally, in Section 4 we present the Yosida-Hewitt decomposition of an order bounded inner regular finitely additive set function.

2. Decomposition of a positive measure. For the basic theory of orthoalgebras needed in this work, the reader is referred to [12], [20] and [22].

The terminology and notation on partially ordered sets, ordered groups and ordered topological groups collected in Section 2 of [7] will be used implicitly in the remainder of this paper. In order to complete the list of ordered topological groups given there, we indicate the non-commutative examples 4.6(4), 4.6(5) and 4.6(7) of [20].

The unexplained terminology and properties concerning lattice groupscalled also $l$-groups - can be found in [4] or [5].

Throughout this section, $L=(L, \perp, \oplus, 0,1)$ is an orthoalgebra and $G=$ $(G, \cdot, e, \leq, \tau)$ is an ordered Hausdorff topological group.

A subset $K$ of $L$ is called a $\delta$-paving in $L$ if the following conditions are satisfied:

(a) $0 \in K$.

(b) Every finite subset of $K$ has a supremum in $(L, \leq)$ which belongs to $K$.

(c) Every countable subset of $K$ has an infimum in $(L, \leq)$ which belongs to $K$.

EXAMPLES 2.1. (1) Let $\Omega$ be a non-empty set, let $L=2^{\Omega}$ be the Boolean algebra of all subsets of $\Omega$ and let $K$ be a $\delta$-ring of subsets of $\Omega$. Then $K$ is a $\delta$-paving in $L$.

(2) For a non-empty set $X$, let $(X, \mathcal{F})$ be a space in the sense of Aleksandrov $\left[1\right.$, p. 314] and let $L$ be the Boolean subalgebra of $2^{X}$ generated by $\mathcal{F}$. Then $\mathcal{F}$ is a $\delta$-paving in $L$. 
(3) Let $\tau$ be the usual topology on $\mathbb{R}$, let $X=[0,1]$ be a subspace of $(\mathbb{R}, \tau)$, let $L=2^{X}$ and let $K$ be the set of all Souslin sets in $X$. Then $K$ is a $\delta$-paving in $L$.

(4) Let $(X, \tau)$ be a Hausdorff topological space, let $L=2^{X}$ and let $K$ be the set of all compact subsets of $X$. Then $K$ is a $\delta$-paving in $L$.

(5) Let $(X, \tau)$ be a locally compact Hausdorff space, let $L=2^{X}$ and let $K$ be the set of all compact $G_{\delta}$-sets in $X$. Then $K$ is a $\delta$-paving in $L$.

(6) Let $H$ be an infinite-dimensional Hilbert space over $\mathbb{C}$, let $A$ be a von Neumann algebra acting on $H$, let $P(A)$ be the orthomodular lattice of all projections in $A$, let $L$ be the orthoalgebra determined by $P(A)$ and let $K=\{e \in L: e$ has a finite-dimensional range $\}$. Then $K$ is a $\delta$-paving in $L$.

For every $a \in L$, let $G_{a}=(G, \cdot, e)$ and let $G^{L}$ be the direct product of the family $\left(G_{a}\right)_{a \in L}$. Then $G^{L}$ ordered by the canonical order $\leq$ induced by $\leq$ is an ordered group.

An element $\mu$ of $G^{L}$ is said to be a measure on $L$ (with values in $G$ ) if $a, b \in G$ and $a \perp b$ imply $\mu(a \oplus b)=\mu(a) \cdot \mu(b)$. For example, if we denote by $e$ the identity element of the group $G^{L}$, then $e$ is a measure on $L$. Henceforth, the set of all measures on $L$ (with values in $G$ ) will be denoted by $a(L, G)$.

Let $\mu$ be an element of $a(L, G)$. Then the following properties are immediate:

(i) $\mu(a) \cdot \mu(b)=\mu(b) \cdot \mu(a)$ whenever $a, b \in L$ and $a \perp b$.

(ii) $\mu(0)=e$.

(iii) If $a, b \in G$ and $a \leq b$, then $\mu(b-a)=\mu(b) \cdot \mu(a)^{-1}=\mu(a)^{-1} \cdot \mu(b)$.

(iv) If $n \in \mathbb{N} \backslash\{0,1\}$ and $\left(a_{i}\right)_{0 \leq i \leq n}$ is a finite orthogonal sequence in $L \backslash\{0\}$, then $\mu\left(\bigoplus_{i=0}^{n} a_{i}\right)=\prod_{i=0}^{n} \mu\left(a_{i}\right)$.

Let $\mu$ be an element of $G^{L}$ such that $\mu(0)=e$. We say that

(a) $\mu$ is positive if $\mu \geq e$.

(b) $\mu$ is countably additive if, for every orthogonal sequence $\left(a_{i}\right)_{i \in \mathbb{N}}$ in $L$ such that $\bigoplus_{i \in \mathbb{N}} a_{i}$ exists in $(L, \leq)$, we have

$$
\mu\left(\bigoplus_{i \in \mathbb{N}} a_{i}\right)=\tau-\lim _{n \rightarrow \infty} \prod_{i=0}^{n} \mu\left(a_{i}\right) .
$$

(c) $\mu$ is inner regular if there exists a $\delta$-paving $K$ in $L$ with the following property: For every $c \in L$ and every $U \in \mathcal{N}(e)$ there exists $b \in K$ such that $b \leq c$ and $\mu(d) \in U$ whenever $d \in L$ and $d \leq c-b$. Sometimes, for more precision, we say that $\mu$ is $K$-inner regular.

(v) Let $\mu$ be a positive element of $a(L, G)$. If $a, b \in L$ and $a \leq b$, then $\mu(a) \leq \mu(b)$. 
If $K$ is a $\delta$-paving in $L$, we denote by $\operatorname{ra}_{K}(L, G)$ the set of all $K$-inner regular measures on $L$ (with values in $G$ ).

Lemma 2.2. Let $K$ be a $\delta$-paving in $L$ and let $\mu_{1}, \mu_{2} \in \operatorname{ra}_{K}(L, G)$. Then

(a) $\mu_{1}^{-1} \in \operatorname{ra}_{K}(L, G)$.

(b) $\mu_{1} \cdot \mu_{2} \in \operatorname{ra}_{K}(L, G)$.

Proof. The statement (a) is trivial. It is also clear that $\mu=\mu_{1} \cdot \mu_{2}$ is an element of $a(L, G)$. Let $c \in L$ and let $U \in \mathcal{N}(e)$. We can choose $V \in \mathcal{N}(e)$ such that $V \cdot V \subseteq U$. Then there exist $b_{1}, b_{2} \in K$ such that $b_{i} \leq c$ and $\mu_{i}(d) \in V$ whenever $d \in L$ and $d \leq c-b_{i}$ for $i=1,2$. Since $K$ is a $\delta$-paving in $L$, it follows that $b_{1} \vee b_{2}$ exists in $(L, \leq)$ and belongs to $K$. Put $b=b_{1} \vee b_{2}$. Let $d \in L$ be such that $d \leq c-b$. Since $b_{i} \leq b \leq c(i=1,2)$, it follows from [20, property iv, p. 196] that $c-b \leq c-b_{i}$. Then $\mu(d)=\mu_{1}(d) \cdot \mu_{2}(d) \in V \cdot V \subseteq U$.

ExAmples 2.3. (1) Let $A=(A,+, \cdot, 0)$ be the ring with unity 1 of all $2 \times 2$ matrices with coefficients in $\mathbb{R}$ and let $*$ be the involution on $A$ given by the transposition. Then $(A, *)$ is a Baer $*$-ring such that the set $P(A)$ of all projections in $A$ consists of 0,1 and all matrices of the form $\left(\begin{array}{ll}x & y \\ y & 1-x\end{array}\right)$ with $x, y \in \mathbb{R}$ and $x^{2}+y^{2}=x$. Since $P(A)$ is an orthomodular lattice (see [19] or $[20]$ ), it determines an orthoalgebra $L$. Let $G$ be the usual ordered additive topological group of real numbers. For $f=\left(\begin{array}{cc}x & y \\ y & 1-x\end{array}\right)$ in $L$, put $\mu(f)=x$. Then $\mu$ is a positive element of $a(L, G)$.

(2) Let $X$ be a Polish space, let $\left(x_{n}\right)_{n \in \mathbb{N}}$ be a sequence of elements of $X$, let $L=\mathcal{B}(X)$ be the $\sigma$-complete Boolean subalgebra of $2^{X}$ of Borel sets in $X$ and let $G$ be the additive lattice topological group derived from the Banach lattice $\left(c_{0}(\mathbb{N}), \leq,\|\cdot\|_{\infty}\right)$. For every $B \in L$, put $\mu(B)=\left(2^{-n} \delta_{x_{n}}(B)\right)_{n \in \mathbb{N}}$ where $\delta_{x_{n}}$ denotes the Dirac measure on $L$ concentrated at $x_{n}$. Then $\mu$ is a positive countably additive element of $\operatorname{ra}_{K}(L, G)$, where $K$ is the set of all compact subsets of $X$.

(3) Let $(\mathbb{C}, V,\langle\cdot, \cdot\rangle)$ be the quadratic space of $[20$, Example 4, p. 200] and let $\mathcal{L}_{S}(V)=\left\{M: M\right.$ is a linear subspace of $V$ and $\left.V=M+M^{\perp}\right\}$. Then $\left(\mathcal{L}_{S}(V), \subseteq,{ }^{\perp}, 0, V\right)$ is an orthomodular poset (see [8] or [20]). Let $L$ be the orthoalgebra derived from $\mathcal{L}_{S}(V)$ and let $G$ be the usual ordered additive topological group of real numbers. Let $f$ be a fixed vector of $V$. Then, for every $M \in L$, there exists a unique decomposition $f=f_{M}+f_{M^{\perp}}$ with $f_{M} \in M$ and $f_{M^{\perp}} \in M^{\perp}$. For every $M \in L$, put $\mu(M)=\int_{\mathbb{R}}\left|f_{M}(x)\right|^{2} d x$. Then $\mu$ is a positive element of $a(L, G)$ which is not countably additive (see $[8])$.

(4) Let $H$ be an infinite-dimensional Hilbert space over $\mathbb{C}$, let $A=B(H)$ be the von Neumann algebra acting on $H$ of all bounded linear operators in $H$, let $\lambda$ be a positive linear form on $A$, let $L$ be the orthoalgebra determined 
by $P(A)$ and let $G$ be the usual ordered multiplicative topological group of non-zero real numbers. For $f \in L$, put $\mu(f)=\exp (\lambda(f))$. Then $\mu$ is a positive element of $a(L, G)$. Moreover, if $\lambda$ is normal, then $\mu$ is countably additive and $K$-inner regular, where $K=\{f \in L: f$ has a finite-dimensional range $\}$ (see [13]).

(5) Let $L=2^{\mathbb{R}}$ and let $I=\mathbb{R}_{+}$. For every $i \in I$, let $G_{i}$ be the additive group of integers ordered by $P=\mathbb{N}$ and endowed with the discrete topology $\tau_{i}$ on $\mathbb{Z}$, let $[i]$ denote the integer part of $i$ and let $J(i)$ be the order interval $[-[i],[i]]$ in $\left(G_{i}, \leq_{\mathbb{N}}\right)$. Let $G$ be the direct product of the groups $G_{i}$, let $\leq$ be the canonical order on $G$ induced by $\leq_{\mathbb{N}}$ and let $\tau$ be the product topology of the topologies $\tau_{i}$. Then $G=\left(\mathbb{Z}^{I},+, 0, \leq, \tau\right)$ is an ordered Hausdorff topological group. For every $A \in L$, put $\mu(A)=(\operatorname{card}(A \cap J(i)))_{i \in I}$. Then $\mu$ is a positive countably additive element of $a(L, G)$.

Let $\mu$ be a positive element of $a(L, G)$ and let $K$ be a $\delta$-paving in $L$. We say that

(a) $\mu$ is $K$-smooth if, for every subset $D$ of $K \backslash\{0\}$ such that $D \downarrow 0$ in $(K \backslash\{0\}, \leq)$, we have $\tau$ - $\lim _{D} \mu(d)=e$.

(b) $\mu$ is $K$-singular if $\mu$ is $K$-inner regular and, for every positive $K$ smooth element $\gamma$ of $\operatorname{ra}_{K}(L, G)$ such that $\gamma \leq \mu$, we have $\gamma=e$.

For example, $e$ is the only positive element of $\operatorname{ra}_{K}(L, G)$ which is $K$ smooth and $K$-singular. The positive measure of Example 2.3(2) is $K$ smooth. Also, if $K$ is any $\delta$-ring of subsets of $\mathbb{R}$, then the positive measure of Example 2.3(5) is $K$-smooth.

It is clear that if $K$ is a $\delta$-paving in $L$ and $\mu_{1}, \mu_{2}$ are two positive elements of $a(L, G)$ which are $K$-smooth, then $\mu_{1} \cdot \mu_{2}$ is also $K$-smooth.

Lemma 2.4. Assume that $K$ is a $\delta$-paving in $L$ and $G$ is locally order convex. Let $\gamma_{1}$ and $\gamma_{2}$ be two positive elements of $a(L, G)$ such that $\gamma_{1} \leq \gamma_{2}$. If $\gamma_{2}$ is $K$-inner regular, then so is $\gamma_{1}$.

Proof. Let $c \in L$ and let $U \in \mathcal{N}(e)$. Since $G$ is locally order convex, it follows from [7, Lemma 2.3] that there exists $V \in \mathcal{N}(e)$ such that $e \leq x \leq y$ and $y \in V$ imply $x \in U$. By the $K$-inner regularity of $\gamma_{2}$, there exists $b \in K$ such that $b \leq c$ and $\gamma_{2}(d) \in V$ whenever $d \in L$ and $d \leq c-b$. Since $\gamma_{1}$ is positive and $\gamma_{1} \leq \gamma_{2}$, it follows that $\gamma_{1}(d) \in U$ whenever $d \in L$ and $d \leq c-b$.

LEMmA 2.5. Assume that $K$ is a $\delta$-paving in $L$ and $G$ is locally order convex. Let $\gamma_{1}$ and $\gamma_{2}$ be two positive elements of a $(L, G)$ such that $\gamma_{1} \leq \gamma_{2}$. If $\gamma_{2}$ is $K$-smooth, then so is $\gamma_{1}$.

Proof. Let $D$ be a subset of $K \backslash\{0\}$ such that $D \downarrow 0$ in $(K \backslash\{0\}, \leq)$ and let $U \in \mathcal{N}(e)$. Then there exists $V \in \mathcal{N}(e)$ such that $e \leq x \leq y$ and $y \in V$ imply $x \in U$. Since $\gamma_{2}$ is $K$-smooth, there exists $d_{0} \in D$ such that $d \in D$ 
and $d \leq d_{0}$ imply $\gamma_{2}(d) \in V$. Then the positivity of $\gamma_{1}$ and the inequality $\gamma_{1} \leq \gamma_{2}$ imply $\gamma_{1}(d) \in U$ whenever $d \in D$ and $d \leq d_{0}$. So $\tau$ - $\lim _{D} \gamma_{1}(d)=e$.

TheORem 2.6. Assume that $L$ is a Boolean algebra and $G$ is locally order convex. Let $K$ be a $\delta$-paving in $L$ and let $\mu$ be a positive $K$-smooth element of $\operatorname{ra}_{K}(L, G)$. Then $\mu$ is countably additive.

Pr o o f. It suffices to show that $\tau-\lim _{n \rightarrow \infty} \mu\left(c_{n}\right)=e$ for every decreasing sequence $\left(c_{n}\right)_{n \in \mathbb{N}}$ of elements of $L$ such that $\bigwedge_{n \in \mathbb{N}} c_{n}=0$. Let $U \in \mathcal{N}(e)$. Choose $V \in \mathcal{N}(e)$ such that $V \cdot V \subseteq U$. Then there exists $W \in \mathcal{N}(e)$ such that $e \leq x \leq y$ and $y \in W$ imply $x \in V$. Moreover, there exists a sequence $\left(W_{i}\right)_{i \in \mathbb{N}}$ of elements of $\mathcal{N}(e)$ such that $W_{0} \cdot W_{1} \cdot \ldots \cdot W_{n} \subseteq W$ for all $n \in \mathbb{N}$. Using the $K$-inner regularity of $\mu$ and the assumption that $L$ is a Boolean algebra, we can construct inductively a sequence $\left(b_{i}\right)_{i \in \mathbb{N}}$ of elements of $K$ such that $b_{i} \leq c_{i} \wedge b_{i-1}$ and $\mu\left(c_{i} \wedge b_{i-1} \wedge b_{i}^{\prime}\right) \in W_{i}$ for all $i \in \mathbb{N}$, where $b_{-1}=1$. We may suppose that $b_{i} \neq 0$ for all $i \in \mathbb{N}$. Then $D=\left\{b_{i}: i \in \mathbb{N}\right\}$ is a downwards filtering subset of $K \backslash\{0\}$ such that $D \downarrow 0$ in $(K \backslash\{0\}, \leq)$. By the $K$-smoothness of $\mu$, we have $\tau$ - $\lim _{i \rightarrow \infty} \mu\left(b_{i}\right)=e$. Since $c_{n}-b_{n}=c_{n} \wedge b_{n}^{\prime}=c_{n} \wedge\left(\bigvee_{i=0}^{n}\left(b_{i-1} \wedge b_{i}^{\prime}\right)\right)=\bigvee_{i=0}^{n}\left(c_{n} \wedge b_{i-1} \wedge b_{i}^{\prime}\right) \leq$ $\bigvee_{i=0}^{n}\left(c_{i} \wedge b_{i-1} \wedge b_{i}^{\prime}\right),\left(c_{i} \wedge b_{i-1} \wedge b_{i}^{\prime}\right)_{i \in \mathbb{N}}$ is an orthogonal sequence in $L$ and $\mu$ is a positive element of $a(L, G)$, it follows from properties (iii)-(v) that $e \leq \mu\left(b_{n}\right)^{-1} \cdot \mu\left(c_{n}\right) \leq \prod_{i=0}^{n} \mu\left(a_{i} \wedge b_{i-1} \wedge b_{i}^{\prime}\right) \in W_{0} \cdot W_{1} \cdot \ldots \cdot W_{n} \subseteq W$ for all $n \in \mathbb{N}$, and therefore $\mu\left(b_{n}\right)^{-1} \cdot \mu\left(c_{n}\right) \in V$ for all $n \in \mathbb{N}$.

Choose $n_{0} \in \mathbb{N}$ such that $n \in \mathbb{N}$ and $n \geq n_{0}$ imply $\mu\left(b_{n}\right) \in V$. Then $\mu\left(c_{n}\right)=\mu\left(b_{n}\right) \cdot\left(\mu\left(b_{n}\right)^{-1} \cdot \mu\left(c_{n}\right)\right) \in V \cdot V \subseteq U$ whenever $n \geq n_{0}$.

Theorem 2.7. Assume that $K$ is a $\delta$-paving in $L$ and $G$ is quasi-order complete, locally order convex and has the property (oc). Then, for every positive element $\mu$ of $\operatorname{ra}_{K}(L, G)$, there exist two positive elements $\xi$ and $\eta$ of $\operatorname{ra}_{K}(L, G)$ with the following properties:

(a) $\mu=\xi \cdot \eta$.

(b) $\xi$ is $K$-smooth.

(c) $\eta$ is K-singular.

Proof. Set $\Gamma=\left\{\gamma \in \operatorname{ra}_{K}(L, G): \gamma\right.$ is positive, $K$-smooth and $\left.\gamma \leq \mu\right\}$. Since $e \in \Gamma, \Gamma$ is non-empty. Let $\Gamma_{0}$ be a totally ordered subset of $(\Gamma, \leq)$.

Let $c \in L$. Then $D(c)=\left\{\gamma(c): \gamma \in \Gamma_{0}\right\}$ is a majorized, upwards filtering subset of $G$. Since $G$ is quasi-order complete, $\bigvee D(c)$ exists in $G$. Write $\gamma_{0}(c)=\bigvee D(c)$ for all $c \in L$. Clearly, $\gamma_{0}$ is a positive element of $G^{L}$ such that $\gamma_{0} \leq \mu$ and, in particular, $\gamma_{0}(0)=e$. Since $\left(\Gamma_{0}, \geq\right)$ is a directed set, $(\gamma(c))_{\gamma \in\left(\Gamma_{0}, \geq\right)}$ is an increasing net in $G$ such that $\bigvee_{\gamma \in \Gamma_{0}} \gamma(c)=\gamma_{0}(c)$ for all $c \in L$. Since $G$ has the property (oc), it follows from [7, Lemma 3.7] that

$$
\gamma_{0}(c)=\tau-\lim _{\Gamma_{0}} \gamma(c) \quad \text { for all } c \in L .
$$


We show that $\gamma_{0}$ is a measure on $L$. Let $a, b \in L$ be such that $a \perp b$. By (1) we have

$$
\begin{aligned}
\gamma_{0}(a \oplus b) & =\tau-\lim _{\Gamma_{0}} \gamma(a \oplus b)=\tau-\lim _{\Gamma_{0}} \gamma(a) \cdot \gamma(b) \\
& =\tau-\lim _{\Gamma_{0}} \gamma(a) \cdot \tau-\lim _{\Gamma_{0}} \gamma(b)=\gamma_{0}(a) \cdot \gamma_{0}(b) .
\end{aligned}
$$

In particular, $\gamma_{0}(1)=\gamma_{0}\left(c \oplus c^{\prime}\right)=\gamma_{0}(c) \cdot \gamma_{0}\left(c^{\prime}\right)$ for all $c \in L$.

We show that

$$
\gamma_{0}(c)=\tau \text { - } \lim _{\Gamma_{0}} \gamma(c) \quad \text { uniformly for } c \in L .
$$

Let $U \in \mathcal{N}(e)$. Since $G$ is locally order convex, there exists a symmetric order convex neighbourhood $V$ of $e$ contained in $U$. By (1), $\gamma_{0}(1)=$ $\tau$ - $\lim _{\Gamma_{0}} \gamma(1)$. So there exists $\gamma_{1} \in \Gamma_{0}$ such that $\gamma \in \Gamma_{0}$ and $\gamma \geq \gamma_{1}$ imply $\gamma(1) \in V \cdot \gamma_{0}(1)$ and therefore $\gamma_{0}(1) \cdot \gamma(1)^{-1} \in V$. Let $c \in L$. Since $\gamma(c) \leq \gamma_{0}(c)$ and $\gamma\left(c^{\prime}\right) \leq \gamma_{0}\left(c^{\prime}\right)$ for all $\gamma \in \Gamma_{0}$, we have $e \leq \gamma_{0}(c) \cdot \gamma(c)^{-1}=$ $\gamma_{0}(1) \cdot \gamma_{0}\left(c^{\prime}\right)^{-1} \cdot \gamma\left(c^{\prime}\right) \cdot \gamma(1)^{-1} \leq \gamma_{0}(1) \cdot \gamma(1)^{-1}$ for all $\gamma \in \Gamma_{0}$. Then $\gamma \in \Gamma_{0}$ and $\gamma \geq \gamma_{1}$ imply $\gamma_{0}(c) \cdot \gamma(c)^{-1} \in V$ and therefore $\gamma(c) \in U \cdot \gamma_{0}(c)$ for all $c \in L$.

We show that $\gamma_{0}$ is $K$-inner regular. Let $c \in L$ and let $U \in \mathcal{N}(e)$. Choose $V \in \mathcal{N}(e)$ such that $V^{-1} \cdot V \subseteq U$. By (2) there exists $\gamma \in \Gamma_{0}$ such that $\gamma(d) \in V \cdot \gamma_{0}(d)$ for all $d \in L$. Since $\gamma$ is $K$-inner regular, there exists $b \in K$ such that $b \leq c$ and $\gamma(d) \in V$ whenever $d \in L$ and $d \leq c-b$. Then $\gamma_{0}(d)=\left(\gamma_{0}(d) \cdot \gamma(d)^{-1}\right) \cdot \gamma(d) \in V^{-1} \cdot V \subseteq U$ whenever $d \in L$ and $d \leq c-b$.

To show that $\gamma_{0}$ is an upper bound of $\Gamma_{0}$ in $(\Gamma, \leq)$, it remains to show that $\gamma_{0}$ is $K$-smooth. Let $D$ be a downwards filtering subset of $K \backslash\{0\}$ such that $D \downarrow 0$ in $(K \backslash\{0\}, \leq)$. Let $U \in \mathcal{N}(e)$. Choose an element $V$ of $\mathcal{N}(e)$ such that $V^{-1} \cdot V \subseteq U$. Then, by (2), there exists $\gamma \in \Gamma_{0}$ such that $\gamma(d) \in V \cdot \gamma_{0}(d)$ for all $d \in L$. Since $\gamma$ is $K$-smooth, there exists $d_{0} \in D$ such that $d \in D$ and $d \leq d_{0}$ imply $\gamma(d) \in V$. Then $\gamma_{0}(d)=\left(\gamma_{0}(d) \cdot \gamma(d)^{-1}\right) \cdot \gamma(d) \in V^{-1} \cdot V \subseteq U$ whenever $d \in L$ and $d \leq d_{0}$.

By the Zorn Lemma, the partially ordered set $(\Gamma, \leq)$ contains a maximal element $\xi$. Then $\xi$ is a positive $K$-smooth element of $\operatorname{ra}_{K}(L, G)$ such that $\xi \leq \mu$. Let $\eta=\xi^{-1} \cdot \mu$. Clearly, $\eta$ is a positive element of $G^{L}$ and $\mu=\xi \cdot \eta$. By Lemma 2.2, $\eta$ is an element of $\operatorname{ra}_{K}(L, G)$. It remains to show that $\eta$ is $K$-singular. Let $\gamma$ be a positive $K$-smooth element of $\operatorname{ra}_{K}(L, G)$ such that $\gamma \leq \eta$. Then $\xi \cdot \gamma \leq \mu$ and, by Lemma $2.2, \xi \cdot \gamma$ is a positive element of $\operatorname{ra}_{K}(L, G)$ which is $K$-smooth. Then $\xi \cdot \gamma \in \Gamma$, and the maximality of $\xi$ implies that $\xi \cdot \gamma=\xi$, and therefore $\gamma=e$.

REMARK 2.8. In the same way we can show that $\eta_{1}=\mu \cdot \xi^{-1}$ is a positive element of $\operatorname{ra}_{K}(L, G)$ which is $K$-singular. Since $\mu=\eta_{1} \cdot \xi$, we get a "right" decomposition of $\mu$. 
Assume that $H=(H, \cdot, e, \leq)$ is a lattice group. The following properties will be used in proving the subsequent decomposition theorems:

(vi) Let $z \in H$ be such that $z=x \cdot y^{-1}$ where $x$ and $y$ are positive elements of $H$. Then $z^{+} \leq x$ and $z^{-} \leq y$.

(vii) Let $x, y \in H$. Then $\left(x \cdot y^{-1}\right)^{+} \leq|x| \cdot|y|$ and $\left(x \cdot y^{-1}\right)^{-} \leq|y| \cdot|x|$.

We denote by ba $(L, G)$ the set of all order bounded elements of $a(L, G)$. For example, if $\mu$ is a positive element of $a(L, G)$, then $\mu \in \mathrm{ba}(L, G)$. Clearly, $\mathrm{ba}(L, G)$ is an ordered subgroup of $(a(L, G), \leq)$ and, moreover, if $G$ is order complete, then $\bigvee_{a \in L} \mu(a)$ and $\bigwedge_{a \in L} \mu(a)$ exist in $G$ for all $\mu \in$ ba $(L, G)$ (see $[17$, Theorem 9, p. 14]).

Lemma 2.9. Assume that $L$ is a Boolean algebra and $G$ is order complete. Then $(\mathrm{ba}(L, G), \cdot, e, \leq)$ is a lattice group.

Proof. Using [5, Lemma 1, p. 292] it suffices to show that, for every $\mu \in b a(L, G)$, there exists $\mu \vee e$ in ba $(L, G)$.

Define $\nu(a)=\bigvee\{\mu(b): b \in L$ and $b \leq a\}$ for all $a \in L$. Then $\nu$ is a positive element of $G^{L}$. We shall show that $\nu$ is an element of $a(L, G)$. Let $c, d \in L$ be such that $c \perp d$. Using the assumption that $L$ is a Boolean algebra, we can deduce the following property:

$b \in L$ and $b \leq c \oplus d \Leftrightarrow$ there exist $c_{1}, d_{1} \in L$ such that $c_{1} \leq c, d_{1} \leq d$ and $b=c_{1} \oplus d_{1}$.

From this and the fact that $\mu \in a(L, G)$, we get

$$
\begin{aligned}
\nu(c \oplus d) & =\bigvee\{\mu(b): b \in L \text { and } b \leq c \oplus d\} \\
& =\bigvee\left\{\mu\left(c_{1} \oplus d_{1}\right): c_{1}, d_{1} \in L, c_{1} \leq c \text { and } d_{1} \leq d\right\} \\
& =\bigvee\left\{\mu\left(c_{1}\right) \cdot \mu\left(d_{1}\right): c_{1}, d_{1} \in L, c_{1} \leq c \text { and } d_{1} \leq d\right\} \\
& =\bigvee\left\{\mu\left(c_{1}\right): c_{1} \in L \text { and } c_{1} \leq c\right\} \cdot \bigvee\left\{\mu\left(d_{1}\right): d_{1} \in L \text { and } d_{1} \leq d\right\} \\
& =\nu(c) \cdot \nu(d) .
\end{aligned}
$$

It remains to show that $\nu=\mu \vee e$ in ba $(L, G)$. Clearly, $\nu$ is an upper bound of the set $\{\mu, e\}$ in $(\mathrm{ba}(L, G), \cdot, e, \leq)$. Let $\lambda$ be an upper bound of the set $\{\mu, e\}$ in $(\mathrm{ba}(L, G), \cdot, e, \leq)$. Then $\lambda \geq e$ and $\lambda \geq \mu$. Let $a \in L$. Then, for all $b \in L$ such that $b \leq a$, we have, by property (v), $\lambda(a) \geq \lambda(b) \geq \mu(b)$, and therefore $\lambda(a) \geq \nu(a)$.

Corollary 2.10. Assume that $L$ is a Boolean algebra and $G$ is order complete. If $\mu \in \mathrm{ba}(L, G)$, then $\mu^{+}$and $\mu^{-}$are given respectively by the formulae:

(a) $\mu^{+}(a)=\bigvee\{\mu(b): b \in L$ and $b \leq a\}$

(b) $\mu^{-}(a)=(\bigwedge\{\mu(b): b \in L \text { and } b \leq a\})^{-1}$ for all $a \in L$. 
Proof. (a) follows from the proof of Lemma 2.9 and (b) follows from (a), [4, formula 1.3.2] and [5, formula (11), p. 292].

First Decomposition Theorem. Assume that $L$ is a Boolean algebra, $K$ is a $\delta$-paving in $L$ and $G$ is order complete, locally order convex and has the property (oc). Then, for every positive element $\mu$ of $\operatorname{ra}_{K}(L, G)$, there exist exactly two positive elements $\xi$ and $\eta$ of $\operatorname{ra}_{K}(L, G)$ with the following properties:

(a) $\mu=\xi \cdot \eta$.

(b) $\xi$ is $K$-smooth.

(c) $\eta$ is K-singular.

Proof. The existence of the decomposition of $\mu$ follows from Theorem 2.7.

To show the uniqueness, suppose that there exist four positive elements $\xi_{1}, \xi_{2}, \eta_{1}$ and $\eta_{2}$ of $\operatorname{ra}_{K}(L, G)$ such that $\xi_{1}$ and $\xi_{2}$ are $K$-smooth, $\eta_{1}$ and $\eta_{2}$ are $K$-singular, $\mu=\xi_{1} \cdot \eta_{1}$ and $\mu=\xi_{2} \cdot \eta_{2}$. Then $\xi_{2}^{-1} \cdot \xi_{1}=\eta_{2} \cdot \eta_{1}^{-1}$. From Lemma 2.9 it follows that $(\mathrm{ba}(L, G), \cdot, e, \leq)$ is a lattice group. Then $\xi_{2}^{-1} \cdot \xi_{1}$ belongs to ba $(L, G)$ and from (vi) it follows that $\left(\xi_{2}^{-1} \cdot \xi_{1}\right)^{+}=\left(\eta_{2} \cdot \eta_{1}^{-1}\right)^{+} \leq \eta_{2}$ and $\left(\xi_{2}^{-1} \cdot \xi_{1}\right)^{-}=\left(\eta_{2} \cdot \eta_{1}^{-1}\right)^{-} \leq \eta_{1}$. Hence, Lemma 2.4 implies that $\left(\xi_{2}^{-1} \cdot \xi_{1}\right)^{+}$ and $\left(\xi_{2}^{-1} \cdot \xi_{1}\right)^{-}$are $K$-inner regular. Moreover, from (vii) it follows that $\left(\xi_{2}^{-1} \cdot \xi_{1}\right)^{+} \leq\left|\xi_{2}^{-1}\right| \cdot\left|\xi_{1}^{-1}\right|=\xi_{2} \cdot \xi_{1}$ and $\left(\xi_{2}^{-1} \cdot \xi_{1}\right)^{-} \leq\left|\xi_{1}^{-1}\right| \cdot\left|\xi_{2}^{-1}\right|=\xi_{1} \cdot \xi_{2}$. Then, by Lemma $2.5,\left(\xi_{2}^{-1} \cdot \xi_{1}\right)^{+}$and $\left(\xi_{2}^{-1} \cdot \xi_{1}\right)^{-}$are $K$-smooth. Since $\eta_{2}$ and $\eta_{1}$ are $K$-singular, it follows that $\left(\xi_{2}^{-1} \cdot \xi_{1}\right)^{+}=e=\left(\xi_{2}^{-1} \cdot \xi_{1}\right)^{-}$. Then [4, formula 1.3.3] implies that $\xi_{2}^{-1} \cdot \xi_{1}=e$. Hence $\xi_{1}=\xi_{2}$ and therefore $\eta_{1}=\eta_{2}$.

The following result will be used in Section 3:

LEMMA 2.11. Assume that $L$ is a Boolean algebra and $G$ is order complete and locally order convex. Let $K$ be a $\delta$-paving in $L$. If $\eta_{1}$ and $\eta_{2}$ are two positive elements of $\operatorname{ra}_{K}(L, G)$ which are $K$-singular, then $\eta_{1} \cdot \eta_{2}$ is also $K$-singular.

Proof. Let $\gamma$ be a positive $K$-smooth element of $\operatorname{ra}_{K}(L, G)$ such that $\gamma \leq \eta_{1} \cdot \eta_{2}$. By Lemma 2.9 it follows that (ba $\left.(L, G), \cdot, e, \leq\right)$ is a lattice group. Applying [4, Corollary 1.2.17] to this group, we can write $\gamma=\gamma_{1} \cdot \gamma_{2}$ where $\gamma_{i}$ is a positive element of $a(L, G)$ such that $\gamma_{i} \leq \eta_{i}$ for $i=1,2$. Since $G$ is locally order convex, it follows from Lemma 2.4 that $\gamma_{i}$ is $K$-inner regular $(i=1,2)$. But $\gamma_{i} \leq \gamma$. Then Lemma 2.5 implies that $\gamma_{i}$ is $K$-smooth for $i=1,2$. Since $\eta_{1}$ and $\eta_{2}$ are $K$-singular,we conclude that $\gamma_{1}=\gamma_{2}=e$, and therefore $\gamma=e$.

3. A general Boolean decomposition. In this section we assume that $L$ is a Boolean algebra and $K$ is a $\delta$-paving in $L$. 
If $G=(G, \cdot, e, \leq, \tau)$ is an ordered Hausdorff topological group, we write $\operatorname{rba}_{K}(L, G)=\operatorname{ba}(L, G) \cap \operatorname{ra}_{K}(L, G)$.

Before we formulate the following result appearing in [13], we recall that, by the Iwasawa Theorem (see [5]), every order complete lattice group is commutative:

Lemma 3.1. Let $G=(G,+, 0, \leq, \tau)$ be a Hausdorff commutative topological lattice group which is order complete and has the property (oc). If $V$ is a sublattice of $(G, \leq)$ and $D$ is a non-empty majorized subset of $V$, then $x=\bigvee D$ exists in $G$ and $x \in \bar{V}$.

Proof. The existence of $\bigvee D$ in $G$ follows from the order completeness of $G$. Let $\mathcal{F}(D)$ denote the set of all finite subsets of $D$. Since $x=\bigvee D=$ $\bigvee\{\bigvee F: F \in \mathcal{F}(D)\}$ and $(\bigvee F)_{F \in(\mathcal{F}(D), \supset)}$ is an increasing net in $G$, the property (oc) implies that $x=\tau-\lim _{\mathcal{F}(D)} \bigvee F$. But $V$ is a sublattice of $(G, \leq)$ and $D \subseteq V$. Then $\bigvee F \in V$ for all $F \in \mathcal{F}(D)$. Hence $x \in \bar{V}$.

Following Jameson [15], a topological lattice group $(G, \cdot, e, \leq, \tau)$ satisfies the condition $(\mathrm{M})$ if there exists a base for the neighbourhood system $\mathcal{N}(e)$ consisting of sublattices of $(G, \leq)$. For example, let $(V, \leq)$ be a Riesz space with an Archimedean order unit $u$ and let $\tau$ denote the metric topology on $V$ induced by $(x, y) \rightarrow \varrho_{u}(x-y)$. Then the additive topological lattice group $(V,+, 0, \leq, \tau)$ satisfies the condition $(\mathrm{M})$.

The following result appears also in [13]:

Proposition 3.2. Let $G=(G,+, 0, \leq, \tau)$ be a Hausdorff commutative topological lattice group which is order complete, has the property (oc) and satisfies the condition (M). If $\mu \in \operatorname{rba}_{K}(L, G)$, then $\mu^{+}$and $\mu^{-}$are $K$-inner regular.

Pr o of. Let $c \in L$ and let $U \in \mathcal{N}(0)$. Since $G$ satisfies the condition (M), there exists $V \in \mathcal{N}(0)$ such that $\bar{V} \subseteq U$ and $V$ is a sublattice of $(G, \leq)$. From the $K$-inner regularity of $\mu$, it follows that there exists $b \in K$ such that $b \leq c$ and $\mu(d) \in V$ whenever $d \in L$ and $d \leq c-b$. In particular, $\{\mu(a): a \in L$ and $a \leq d\} \subseteq V$ for all $d \in D$ and $d \leq c-b$.

Since $\mu \in \mathrm{ba}(L, G)$, it follows from Corollary 2.10 and Lemma 3.1 that $\mu^{+}(d)=\bigvee\{\mu(a): a \in L$ and $a \leq d\} \in \bar{V} \subseteq U$ whenever $d \in L$ and $d \leq c-b$.

The $K$-inner regularity of $\mu^{-}$follows from the above result, Lemma 2.2 and [4, formula 1.3.2].

Let $G=(G, \cdot, e, \leq, \tau)$ be an ordered Hausdorff topological group which is order complete and let $\mu \in \mathrm{ba}(L, G)$. We say that

(a) $\mu$ is $K$-smooth if $\mu^{+}$and $\mu^{-}$are $K$-smooth. 
(b) $\mu$ is $K$-singular if $\mu$ is $K$-inner regular and, for every pair $\gamma_{1}$ and $\gamma_{2}$ of positive $K$-smooth elements of $\operatorname{ra}_{K}(L, G)$ such that $\gamma_{1} \leq \mu^{+}$and $\gamma_{2} \leq \mu^{-}$, we have $\gamma_{1}=\gamma_{2}=e$.

Clearly, if $\mu$ is $K$-smooth, then so is $|\mu|$.

Lemma 3.3. Let $G=(G,+, 0, \leq, \tau)$ be a Hausdorff commutative topological lattice group which is order complete, has the property (oc) and satisfies the condition (M). If $\mu \in \operatorname{rba}_{K}(L, G)$ is $K$-smooth and $K$-singular, then $\mu=0$.

Proof. This follows immediately from the definitions and Proposition 3.2 .

The following result generalizes [2, Theorem 6, p. 591]:

TheOREM 3.4. Let $G=(G,+, 0, \leq, \tau)$ be a Hausdorff commutative topological lattice group which is order complete, locally order convex, has the property (oc) and satisfies the condition (M), and let $\mu$ be an element of $\operatorname{rba}_{K}(L, G)$. If $\mu$ is $K$-smooth, then $\mu$ is countably additive.

Proof. By Lemma 2.9 and [4, formula 1.3.3] we can write $\mu=\mu^{+}-\mu^{-}$, where $\mu^{+}$and $\mu^{-}$are positive elements of $a(L, G)$. Moreover, Proposition 3.2 implies that $\mu^{+}$and $\mu^{-}$are $K$-inner regular. Since $\mu$ is $K$-smooth, it follows that so are $\mu^{+}$and $\mu^{-}$. Then, by Theorem $2.6, \mu^{+}$and $\mu^{-}$are countably additive. Therefore $\mu$ is countably additive.

Second Decomposition Theorem. Let $G=(G,+, 0, \leq, \tau)$ be a Hausdorff commutative topological lattice group which is order complete, locally order convex, has the property (oc) and satisfies the condition (M). Then, for every element $\mu$ of $\operatorname{rba}_{K}(L, G)$, there exist exactly two elements $\xi$ and $\eta$ of $\operatorname{rba}_{K}(L, G)$ with the following properties:

(a) $\mu=\xi+\eta$.

(b) $\xi$ is $K$-smooth.

(c) $\eta$ is K-singular.

P r o of. We first show the existence of the decomposition.

From Lemma 2.9, [4, formula 1.3.3] and Proposition 3.2, we can write $\mu=\mu^{+}-\mu^{-}$, where $\mu^{+}$and $\mu^{-}$are positive elements of $\operatorname{ra}_{K}(L, G)$. By the First Decomposition Theorem there exist four positive elements $\xi_{1}, \xi_{2}, \eta_{1}$ and $\eta_{2}$ of $\operatorname{ra}_{K}(L, G)$ such that $\xi_{1}$ and $\xi_{2}$ are $K$-smooth, $\eta_{1}$ and $\eta_{2}$ are $K$ singular, $\mu^{+}=\xi_{1}+\eta_{1}$ and $\mu^{-}=\xi_{2}+\eta_{2}$. Since $G$ is commutative, we have $\mu=\left(\xi_{1}-\xi_{2}\right)+\left(\eta_{1}-\eta_{2}\right)$. Clearly, $\xi_{1}-\xi_{2}, \eta_{1}-\eta_{2} \in \operatorname{ba}(L, G)$, and it follows from Lemma 2.2 that $\xi_{1}-\xi_{2}$ and $\eta_{1}-\eta_{2}$ are $K$-inner regular. So $\xi_{1}-\xi_{2}, \eta_{1}-\eta_{2} \in \operatorname{rba}_{K}(L, G)$. On the other hand, from property (vi) and Lemma 2.5 it follows that $\xi_{1}-\xi_{2}$ is $K$-smooth. Finally, to show that $\eta_{1}-\eta_{2}$ is $K$-singular, let $\gamma_{1}$ and $\gamma_{2}$ be two positive $K$-smooth elements of 
$\operatorname{ra}_{K}(L, G)$ such that $\gamma_{1} \leq\left(\eta_{1}-\eta_{2}\right)^{+}$and $\gamma_{2} \leq\left(\eta_{1}-\eta_{2}\right)^{-}$. By (vii) we have $\left(\eta_{1}-\eta_{2}\right)^{+} \leq \eta_{1}$ and $\left(\eta_{1}-\eta_{2}\right)^{-} \leq \eta_{2}$. Since $\eta_{1}$ and $\eta_{2}$ are $K$-singular, we get $\gamma_{1}=0$ and $\gamma_{2}=0$.

Now we show the uniqueness of the decomposition.

Suppose that there exist $\xi_{1}, \xi_{2}, \eta_{1}, \eta_{2} \in \operatorname{rba}_{K}(L, G)$ such that $\xi_{1}$ and $\xi_{2}$ are $K$-smooth, $\eta_{1}$ and $\eta_{2}$ are $K$-singular and $\mu=\xi_{1}+\eta_{1}=\xi_{2}+\eta_{2}$. Then $\xi_{1}-\xi_{2}=\eta_{2}-\eta_{1}$, and for $i=1,2,\left|\xi_{i}\right|$ and $\left|\eta_{i}\right|$ are positive elements of $\operatorname{ra}_{K}(L, G)$ such that $\left|\xi_{i}\right|$ is $K$-smooth. Since, by (vii), $\left(\xi_{1}-\xi_{2}\right)^{+},\left(\xi_{1}-\xi_{2}\right)^{-} \leq$ $\left|\xi_{1}\right|+\left|\xi_{2}\right|$, it follows from Lemma 2.5 that $\xi_{1}-\xi_{2}$ is $K$-smooth. On the other hand, since $\eta_{i}$ is $K$-singular, Proposition 3.2 implies that $\eta_{i}^{+}$and $\eta_{i}^{-}$are $K$-singular, and therefore, by Lemma 2.11, it follows that $\left|\eta_{i}\right|$ is $K$-singular for $i=1,2$. Again, by Lemma 2.11, $\left|\eta_{1}\right|+\left|\eta_{2}\right|$ is $K$-singular. We show that $\eta_{2}-\eta_{1}$ is $K$-singular. Let $\gamma_{1}$ and $\gamma_{2}$ be two positive $K$-smooth elements of $\operatorname{ra}_{K}(L, G)$ such that $\gamma_{1} \leq\left(\eta_{2}-\eta_{1}\right)^{+}$and $\gamma_{2} \leq\left(\eta_{2}-\eta_{1}\right)^{-}$. Then, by formula (vii), we have $\gamma_{1}, \gamma_{2} \leq\left|\eta_{1}\right|+\left|\eta_{2}\right|$ and therefore $\gamma_{1}=0$ and $\gamma_{2}=0$. So $\xi_{1}-\xi_{2}$ is a $K$-smooth and $K$-singular element of $\operatorname{rba}_{K}(L, G)$. By Lemma $3.3, \xi_{1}-\xi_{2}=0$ and therefore $\xi_{1}=\xi_{2}$. This implies that $\eta_{1}=\eta_{2}$.

REMARK 3.5. Using the results established in [7] it is easy to verify that the Second Decomposition Theorem generalizes the classical first Aleksandrov Decomposition Theorem [2, Theorem 3, pp. 618-619].

4. Yosida-Hewitt decomposition of an inner regular measure. Let $X$ be a non-empty set, let $2^{X}$ be the Boolean algebra of all subsets of $X$ and let $\mathcal{F}$ be a $\delta$-paving in $2^{X}$ containing $X$. Then the pair $(X, \mathcal{F})$ is called, following Aleksandrov [1], a space. The elements of $\mathcal{F}$ are called closed sets in $X$ and their complements are called open sets in $X$. For example, if $(X, \tau)$ is a topological space and $\mathcal{F}=\left\{F \subseteq X: F=f^{-1}(0)\right.$ for some continuous function $f$ from $X$ into $\mathbb{R}\}$, then $(X, \mathcal{F})$ is a space.

A space $(X, \mathcal{F})$ is said to be Lindelöf if every covering of $X$ by open sets in $X$ contains a countable subcovering. For example, if $(X, \tau)$ is a Souslin space and $\mathcal{F}=\{F \subseteq X: X \backslash F \in \tau\}$, then $(X, \mathcal{F})$ is a Lindelöf space.

We note that in $[2$, Theorem 9 , p. 592] it is shown that in every normal and non-bicompact space $(X, \mathcal{F})$ there exists an element $\mu \in \operatorname{rba}_{\mathcal{F}}(L, \mathbb{R})$ which is not $\mathcal{F}$-smooth, where $L$ is the Boolean subalgebra of $2^{X}$ generated by $\mathcal{F}$. Then, considering this element $\mu$, the Second Decomposition Theorem gives a non-trivial decomposition for $\mu$.

Let $L$ be an orthoalgebra, let $G=(G, \cdot, e, \tau)$ be a Hausdorff topological group and let $\mu$ be an element of $G^{L}$ such that $\mu(0)=e$. We say that $\mu$ is $s$-bounded if, for every orthogonal sequence $\left(a_{i}\right)_{i \in \mathbb{N}}$ of elements of $L$, we have $\tau$ - $\lim _{i \rightarrow \infty} \mu\left(a_{i}\right)=e$. For example, the measure given by Example $2.3(3)$ is $s$-bounded. We will denote by $\operatorname{sa}(L, G)$ (resp. ca $(L, G))$ the set of 
all $s$-bounded (resp. countably additive) measures on $L$ (with values in $G$ ). Clearly, if $\mu_{1}, \mu_{2} \in \operatorname{sa}(L, G)$, then $\mu_{1}^{-1}, \mu_{1} \cdot \mu_{2} \in \operatorname{sa}(L, G)$.

LEMMA 4.1. Let $L$ be an orthoalgebra and let $G=(G, \cdot, e, \leq, \tau)$ be an ordered Hausdorff topological group which is quasi-order complete and has the property (oc). If $\mu$ is a positive element of $a(L, G)$, then $\mu$ is s-bounded.

Pr o o f. Let $\left(a_{i}\right)_{i \in \mathbb{N}}$ be an orthogonal sequence in $L$. For every $n \in \mathbb{N}$, put $x_{n}=\prod_{i=0}^{n} \mu\left(a_{i}\right)$. Since $\mu$ is positive, it follows that $\left(x_{n}\right)_{n \in \mathbb{N}}$ is an increasing sequence in $G$. Moreover, the set $\left\{x_{n}: n \in \mathbb{N}\right\}$ is an upwards filtering subset of $G$ majorized by $\mu(1)$. Since $G$ is quasi-order complete, $x=\bigvee_{n \in \mathbb{N}} x_{n}$ exists in $G$. Then, by the property (oc), we get $x=\tau-\lim _{n \rightarrow \infty} x_{n}$. Since $\mu\left(a_{n}\right)=x_{n} \cdot x_{n-1}^{-1}$ with $x_{-1}=e$, it follows that $\tau-\lim _{n \rightarrow \infty} \mu\left(a_{n}\right)=x \cdot x^{-1}=e$.

Corollary 4.2. Let $L$ be a Boolean algebra and let $G=(G, \cdot, e, \leq, \tau)$ be an ordered Hausdorff topological group which is order complete and has the property (oc). Then every element $\mu$ of ba $(L, G)$ is s-bounded.

Proof. This follows immediately from Lemmas 2.9 and 4.1 and [4, formula 1.3.3].

Lemma 4.3. Let $L$ be a Boolean algebra and let $G=(G,+, 0, \leq, \tau)$ be a Hausdorff commutative topological lattice group which is order complete, has the property (oc) and satisfies the condition (M). If $\mu \in \mathrm{ba}(L, G) \cap \mathrm{ca}(L, G)$, then $\mu^{+}$and $\mu^{-}$are countably additive.

Proof. It suffices to show the countable additivity of $\mu^{+}$. Let $\left(c_{n}\right)_{n \in \mathbb{N}}$ be a decreasing sequence of elements of $L$ such that $\bigwedge_{n \in \mathbb{N}} c_{n}=0$. It remains to show that $\tau$ - $\lim _{n \rightarrow \infty} \mu^{+}\left(c_{n}\right)=0$. Let $U \in \mathcal{N}(0)$. Since $G$ satisfies the condition (M), there exists $V \in \mathcal{N}(0)$ such that $\bar{V} \subseteq U$ and $V$ is a sublattice of $(G, \leq)$. By [23, Lemma 1], there exists $n_{0} \in \mathbb{N}$ such that $a \in L$ and $a \leq c_{n_{0}}$ imply $\mu(a) \in V$. So $\left\{\mu(a): a \in L\right.$ and $\left.a \leq c_{n_{0}}\right\} \subseteq V$. Let $n \in \mathbb{N}$ be such that $n \geq n_{0}$. Since $\left\{\mu(a): a \in L\right.$ and $\left.a \leq c_{n}\right\} \subseteq\left\{\mu(a): a \in L\right.$ and $\left.a \leq c_{n_{0}}\right\}$ and $\mu \in \mathrm{ba}(L, G)$, it follows from Lemma 3.1 that $\mu^{+}\left(c_{n}\right)=\bigvee\{\mu(a): a \in L$ and $\left.a \leq c_{n}\right\} \in \bar{V} \subseteq U$, and therefore $\tau$ - $\lim _{n \rightarrow \infty} \mu^{+}\left(c_{n}\right)=0$.

Lemma 4.4. Let $L$ be a Boolean algebra, let $G=(G, \cdot, e, \leq, \tau)$ be an ordered Hausdorff topological group which is locally order convex and let $\mu$ be a positive element of $\mathrm{ca}(L, G)$. If $\gamma$ is a positive element of $a(L, G)$ such that $\gamma \leq \mu$, then $\gamma$ is countably additive.

Proof. Let $\left(c_{n}\right)_{n \in \mathbb{N}}$ be a decreasing sequence of elements of $L$ such that $\bigwedge_{n \in \mathbb{N}} c_{n}=0$. It suffices to show that $\tau-\lim _{n \rightarrow \infty} \gamma\left(c_{n}\right)=e$. Let $U \in$ $\mathcal{N}(e)$. Since $G$ is locally order convex, there exists $V \in \mathcal{N}(e)$ such that $e \leq x \leq y$ and $y \in V$ imply $x \in U$. But $\mu$ is countably additive. Then $\tau-\lim _{n \rightarrow \infty} \mu\left(c_{n}\right)=e$. So there exists $n_{0} \in \mathbb{N}$ such that $n \in \mathbb{N}$ and $n \geq n_{0}$ imply $\mu\left(c_{n}\right) \in V$. Hence $\gamma\left(c_{n}\right) \in U$ whenever $n \geq n_{0}$. 
Let $L$ be an orthoalgebra, let $G=(G, \cdot, e, \leq, \tau)$ be an ordered Hausdorff topological group and let $\mu$ be a positive element of $a(L, G)$. We say that $\mu$ is purely finitely additive if, for every positive element $\gamma$ of ca $(L, G)$ such that $\gamma \leq \mu$, we have $\gamma=e$. For example, let $H$ be a separable infinite-dimensional Hilbert space over $\mathbb{C}$, let $\left(\varphi_{n}\right)_{n \in \mathbb{N}}$ be an orthonormal base of $H$, let $L$ be the orthoalgebra determined by $P(B(H))$ and let LIM denote the Banach limit on $\ell^{\infty}(\mathbb{N})$. For every $f \in L$, put $\mu(f)=\operatorname{LIM}\left(\left(\left\|f \varphi_{n}\right\|\right)_{n \in \mathbb{N}}\right)$. Then it is easy to see that $\mu$ is a positive element of $a(L, \mathbb{R})$ which is purely finitely additive.

Now assume that $L$ is a Boolean algebra and $G$ is order complete. Then an element $\mu$ of $\mathrm{ba}(L, G)$ is said to be purely finitely additive if $\mu^{+}$and $\mu^{-}$are purely finitely additive. For example, let $L=2^{\mathbb{Z}}$, let $M^{+}(\mathbb{Z})=$ $\{\mu \in a(L, \mathbb{R}): \mu$ takes only the values 0 and 1$\}$ and let $M(\mathbb{Z})=\{\mu=$ $\left.\mu_{1}-\mu_{2}: \mu_{1}, \mu_{2} \in M^{+}(\mathbb{Z})\right\}$. We note that an element $\mu \in M^{+}(\mathbb{Z}) \backslash\{0\}$ is countably additive if and only if there exists $k \in \mathbb{Z}$ such that $\mu(\{k\})=1$. Let $\mu=\mu_{1}-\mu_{2}$ be an element of $M(\mathbb{Z})$ such that $\mu_{1}(\{k\})=\mu_{2}(\{k\})=0$ for all $k \in \mathbb{Z}$. Then $\mu$ is purely finitely additive.

Lemma 4.5. Let $L$ be a Boolean algebra, let $G=(G, \cdot, e, \leq, \tau)$ be an ordered Hausdorff topological group which is order complete and locally order convex and let $\mu_{1}$ and $\mu_{2}$ be two positive elements of $a(L, G)$. If $\mu_{1}$ and $\mu_{2}$ are purely finitely additive, then so is $\mu_{1} \cdot \mu_{2}$.

Proof. Let $\gamma$ be a positive element of $\operatorname{ca}(L, G)$ such that $\gamma \leq \mu_{1} \cdot \mu_{2}$. Consider the lattice group (ba $(L, G), \cdot, e, \leq)$. Then, by [4, Corollary 1.2.17] we can write $\gamma=\gamma_{1} \cdot \gamma_{2}$ where $\gamma_{1}$ and $\gamma_{2}$ are positive elements of $a(L, G)$ such that $\gamma_{1} \leq \mu_{1}$ and $\gamma_{2} \leq \mu_{2}$. Since $\gamma_{1}, \gamma_{2} \leq \gamma$, it follows from Lemma 4.4 that $\gamma_{1}$ and $\gamma_{2}$ are countably additive. But $\mu_{1}$ and $\mu_{2}$ are purely finitely additive. Then $\gamma_{1}=e$ and $\gamma_{2}=e$, and therefore $\gamma=e$.

Corollary 4.6. Let $L$ be a Boolean algebra, let $G=(G, \cdot, e, \leq, \tau)$ be an ordered Hausdorff topological group which is order complete and locally order convex and let $\mu$ be an element of $\mathrm{ba}(L, G)$. If $\mu$ is purely finitely additive, then so is $|\mu|$.

Pro of. This follows immediately from the definition, [4, formula 1.3.13] and Lemma 4.5.

We also need the following corrected version of [7, Lemma 4.11]:

LEMMA 4.7. Let $(X, \mathcal{F})$ be a Lindelöf space, let $L$ be a Boolean subalgebra of $2^{X}$ containing $\mathcal{F}$ and let $G=(G, \cdot, e, \tau)$ be a Hausdorff topological group. If $\mu \in \operatorname{sa}(L, G) \cap \operatorname{ca}(L, G)$ then $\mu$ is $\mathcal{F}$-smooth.

We are in a position to establish a Yosida-Hewitt decomposition for an inner regular measure: 
Theorem 4.8. Let $(X, \mathcal{F})$ be a Lindelöf space, let $L$ be a Boolean subalgebra of $2^{X}$ containing $\mathcal{F}$, and let $G=(G,+, 0, \leq, \tau)$ be a Hausdorff commutative topological lattice group which is order complete, locally order convex, has the property (oc) and satisfies the condition (M). Then for every $\mu \in \operatorname{rba}_{\mathcal{F}}(L, G)$, there exist exactly two elements $\xi$ and $\eta$ of $\operatorname{rba}_{\mathcal{F}}(L, G)$ with the following properties:

(a) $\mu=\xi+\eta$.

(b) $\xi$ is countably additive.

(c) $\eta$ is purely finitely additive.

P r o of. We first show the existence of the decomposition.

By the Second Decomposition Theorem there exist $\xi, \eta \in \operatorname{rba}_{\mathcal{F}}(L, G)$ such that $\xi$ is $\mathcal{F}$-smooth, $\eta$ is $\mathcal{F}$-singular and $\mu=\xi+\eta$. By Theorem 3.4, $\xi$ is countably additive. It remains to show that $\eta$ is purely finitely additive. Let $\gamma_{1}$ and $\gamma_{2}$ be two positive elements of ca $(L, G)$ such that $\gamma_{1} \leq \eta^{+}$and $\gamma_{2} \leq \eta^{-}$. Since $\eta \in \operatorname{rba}_{\mathcal{F}}(L, G)$, Proposition 3.2 shows that $\eta^{+}$and $\eta^{-}$are $\mathcal{F}$-inner regular. Then, by Lemma $2.4, \gamma_{1}$ and $\gamma_{2}$ are $\mathcal{F}$-inner regular. On the other hand, Lemma 4.1 implies that $\gamma_{1}$ and $\gamma_{2}$ are $s$-bounded. Since $(X, \mathcal{F})$ is a Lindelöf space and $L$ is a Boolean subalgebra of $2^{X}$ containing $\mathcal{F}$, it follows from Lemma 4.7 that $\gamma_{1}$ and $\gamma_{2}$ are $\mathcal{F}$-smooth. But $\eta$ is $\mathcal{F}$-singular. Hence $\gamma_{1}=0$ and $\gamma_{2}=0$. This implies that $\eta$ is purely finitely additive.

Now we show the uniqueness of the decomposition.

Suppose that there exist $\xi_{1}, \xi_{2}, \eta_{1}, \eta_{2} \in \operatorname{rba}_{\mathcal{F}}(L, G)$ such that $\xi_{1}$ and $\xi_{2}$ are countably additive, $\eta_{1}$ and $\eta_{2}$ are purely finitely additive and $\mu=$ $\xi_{1}+\eta_{1}=\xi_{2}+\eta_{2}$. Then $\xi_{1}-\xi_{2}=\eta_{2}-\eta_{1}$. Since $\xi_{1}-\xi_{2} \in \operatorname{ba}(L, G) \cap \operatorname{ca}(L, G)$, Lemma 4.3 implies that $\left(\xi_{1}-\xi_{2}\right)^{+}$and $\left(\xi_{1}-\xi_{2}\right)^{-}$are countably additive. By (vii) we have $\left(\xi_{1}-\xi_{2}\right)^{+}=\left(\eta_{2}-\eta_{1}\right)^{+} \leq\left|\eta_{1}\right|+\left|\eta_{2}\right|$ and $\left(\xi_{1}-\xi_{2}\right)^{-}=$ $\left(\eta_{2}-\eta_{1}\right)^{-} \leq\left|\eta_{1}\right|+\left|\eta_{2}\right|$. Since $\eta_{1}$ and $\eta_{2}$ are purely finitely additive, Corollary 4.6 and Lemma 4.5 imply that $\left|\eta_{1}\right|+\left|\eta_{2}\right|$ is purely finitely additive as well. Then $\left(\xi_{1}-\xi_{2}\right)^{+}=0$ and $\left(\xi_{1}-\xi_{2}\right)^{-}=0$ and therefore $\xi_{1}=\xi_{2}$. Hence $\eta_{1}=\eta_{2}$.

\section{References}

[1] A. D. Alexandroff [A. D. Aleksandrov], Additive set-functions in abstract spaces, Part 1, Mat. Sb. 8 (50) (1940), 307-348.

[2] - - Additive set-functions in abstract spaces, Part 2, ibid. 9 (51) (1941), 563-628.

[3] E. G. Beltrametti and G. Cassinelli, The Logic of Quantum Mechanics, Addison-Wesley, Reading, Mass., 1981.

[4] A. Bigard, K. Keimel et S. Wolfenstein, Groupes et anneaux réticulés, Lecture Notes in Math. 608, Springer, New York, 1977.

[5] G. Birkhoff, Lattice Theory, Amer. Math. Soc. Colloq. Publ. 25, 3rd ed., Providence, R.I., 1967.

[6] G. Birkhoff and J. von Neumann, The logic of quantum mechanics, Ann. of Math. 37 (1936), 823-843. 
[7] P. De Lucia and P. Morales, Non-commutative version of the Alexandroff Decomposition Theorem in ordered topological groups, preprint no. 51, Univ. of Naples, 1993, $21 \mathrm{pp}$

[8] A. Dvurečenskij, Gleason's Theorem and Its Applications, Kluwer, Dordrecht, 1993.

[9] A. Dvurečenskij and B. Riečan, Decomposition of measures on orthoalgebras and difference posets, Internat. J. Theoret. Phys. 33 (1994), 1387-1402.

[10] D. Feldman and A. Wilce, $\sigma$-Additivity in manuals and orthoalgebras, Order 10 (1993), 383-392.

[11] D. J. Foulis and M. K. Bennett, Tensor product of orthoalgebras, ibid., 271-282.

[12] D. J. Foulis, R. J. Greechie and G. T. Rüttimann, Filters and supports in orthalgebras, Internat. J. Theoret. Phys. 31 (1992), 789-807.

[13] F. Garcia-Mazario, Ordered topological group-valued measures on orthoalgebras, doctoral dissertation, UNED, 1995 (in Spanish).

[14] E. D. Habil, Brooks-Jewett and Nikodym convergence theorems for orthoalgebras that have the weak subsequential property, Internat. J. Theoret. Phys. 34 (1995), 465-491.

[15] G. Jameson, Ordered Linear Spaces, Lecture Notes in Math. 141, Springer, New York, 1970.

[16] G. Kalmbach, Orthomodular Lattices, Academic Press, London, 1983.

[17] J. Kelley, General Topology, Grad. Texts in Math. 27, Springer, New York, 1985.

[18] G. W. Mackey, The Mathematical Foundations of Quantum Mechanics, Benjamin, New York, 1963.

[19] S. Maeda, Probability measures on projections in von Neumann algebras, Rev. Math. Phys. 1 (1990), 235-290.

[20] P. Morales and F. Garcia-Mazario, The support of a measure in ordered topological groups, Atti Sem. Mat. Fis. Univ. Modena 45 (1997), 179-221.

[21] G. T. Rüttimann, Non-commutative measure theory, Habilitationsschrift, Universität Bern, 1980.

[22] —, The approximate Jordan-Hahn decomposition, Canad. J. Math. 41 (1989), 11241146.

[23] K. Sundaresan and P. W. Day, Regularity of group valued Baire and Borel measures, Proc. Amer. Math. Soc. 36 (1972), 609-612.

[24] V. S. Varadarajan, Geometry of Quantum Theory, 2nd ed., Springer, Berlin, 1985 .

[25] K. Yosida and E. Hewitt, Finitely additive measures, Trans. Amer. Math. Soc. 72 (1952), 46-66.

Dipartimento di Matematica

e Applicazioni

Università Federico II

Complesso Universitario Monte S. Angelo

I-80126 Napoli, Italy

E-mail: delucia@matna3.dma.unina.it
Département de mathématiques et d'informatique Université de Sherbrooke Sherbrooke, Québec J1K 2R1

Canada

E-mail: anne.lacasse@dmi.usherb.ca 